\title{
(Re)Using Qualitative Data? ${ }^{[1]}$
}

\author{
by Niamh Moore \\ University of Manchester \\ Sociological Research Online, Volume 12, Issue 3, \\ $<$ http://umw.socresonline.org.uk/12/3/1.html> \\ doi:10.5153/sro. 1496
}

Received: 1 Mar 2006 Accepted: 11 Jan 2007 Published: 30 May 2007

\begin{abstract}
Recent interest by social scientists in the questions posed by reusing qualitative data has been prompted by two related events. The first is the establishment of the Qualitative Data Archival Resource Centre (QUALIDATA, and, since 2003, ESDS Qualidata) at the University of Essex in 1994. The second is the publication of the Economic and Social Research Council (ESRC) Datasets Policy (1996) which asks that those in receipt of ESRC grants offer copies of their data for deposit to QUALIDATA. This perceived injunction to archive data has been met with resistance by recalcitrant researchers who are wary of the implications of depositing data, and the possibilities of reusing data. The debate risks becoming polarised between those advocating the archiving and reuse of qualitative data, and those more sceptical of these possibilities. This paper aims to open up this debate and to seek a more fruitful path between these positions. I begin by calling into question the supposed 'newness' of reusing qualitative data, through turning to examine some of the assumptions embedded in the key terms and premises of the debate thus far, including the reliance on distinctions between primary and secondary data and primary and secondary analysis. I examine some common tropes in accounts of reusing data: comparisons with secondary analysis of quantitative data; efforts to distinguish between reusing qualitative data in a sociological context and other disciplinary and methodological traditions; and reliance on particular interpretations of key principles of qualitative research, context and reflexivity, in establishing the challenges of the reusing of qualitative data. I suggest that reuse may be more productively understood as a process of recontextualising data, and that attending to the reflexive production of data in the contemporary research project may offer more hopeful possibilities for reuse. I conclude by offering some reflections on why discussions of reusing qualitative data appear to have become so fraught.
\end{abstract}

\section{Keywords: ESDS Qualidata, Qualitative Methodology, Reusing Qualitative Data, Secondary Analysis, Secondary Data}

\section{Introduction}

1.1 Recent interest by social scientists in the UK in the questions posed by reusing qualitative data can be traced to two particular events. The first is the establishment of the Qualitative Data Archival Resource Centre (QUALIDATA, and, since 2003, ESDS Qualidata) at the University of Essex in 1994. The second is the publication of the Economic and Social Research Council (ESRC) Datasets Policy (1996) which asks that those in receipt of ESRC grants offer copies of their data for deposit to QUALIDATA. These two events have given rise to a burgeoning literature around the reuse of qualitative data. Discussions have manifested in a variety of fora. Initially they took place in specialised locations. These included ESRC reports on data archiving and policy (see Boddy 2001); publications from those associated with QUALIDATA outlining the possibilities offered by archiving and re-using data (Corti, Foster et al. 1995, Corti and Thompson 1998, Thompson 2004); special issues of the online e-journal Forum Qualitative Socialforschung / Qualitative Research Forum on 'Text, Archive and Re-analysis' (Corti, Kluge et al. 2000, December) and 'Qualitative Inquiry: Research, Archiving, and Reuse' (Bergman and Eberle 2005, May); and a number of Social Research Updates on 'Secondary Analysis of Qualitative Data' (Heaton 1998) and 'Archiving and Re-using Qualitative Data (Corti, Foster et al. 1995). More recently, the publication of the first monograph on the topic in 2004, Reworking Qualitative Data (Heaton 2004a), and a recent debate in the journal Sociology about archiving and re-using data, begin to suggest that the debate is moving into the mainstream of sociological debate (Mauthner et al 1998, Parry and Mauthner 2004, Parry and Mauthner 2005, Bishop 2005a). Yet, rather than signalling a greater acceptance of the idea of reusing qualitative data, the debate risks becoming increasingly polarised. On the one hand are those who are advocates of reusing data (often 
linked directly or indirectly with ESDS Qualidata), and on the other are accounts from those who have embarked on secondary analysis only to find it fraught with more difficulties than perhaps were anticipated (Heaton 2004b); Mauthner et al. have even written of the perceived failure of their projects to reuse existing data (mauthner 1998).

1.2 This paper aims to open up this debate and seeks a more fruitful path between these positions. It does this by calling into question the supposed 'newness' of reusing qualitative data, and unpacking many of the implicit and sometimes explicit assumptions in accounts of reusing qualitative data. This paper calls into question the terms of debate around what has variously been termed 'reusing qualitative data', or 'secondary analysis of qualitative data', in order to pose 'reusing qualitative data' as a question rather than as a taken for granted category. Ultimately the paper questions the 're' of reusing data, asking whether this focus has been productive, and whether in this process of thinking about reusing data, some of the principles of qualitative research, of 'simply' using data, risk being lost. A review of the literature reveals the subjects under discussion, 'reusing qualitative data', 'secondary data' and 'secondary analysis', to be more fragile entities than might be anticipated. Distinctions from 'primary data', 'primary analysis', and 'primary researchers' have been carefully constructed. In producing such differences contributors to this debate rely on secondary analysis of quantitative data as a point of comparison for accounts of reusing qualitative data. Yet this way of framing leaves distinctions between using and reusing qualitative data assumed rather than explored; and marginalises comparisons with other approaches to qualitative data. I focus on the deployment of disciplinary, and other, boundaries in these debates, particularly efforts to make distinctions between disciplines such as sociology and history, and between archives such as ESDS Qualidata and Mass Observation. Then I turn to principles of qualitative research which have been invoked, particularly the importance of context and the role of reflexivity in constructing qualitative data, in order to reinterpret the meaning and practice of the 'qualitative' which connects '(re)using' and 'data'. I conclude by offering some reflections on how and why this debate appears to have become so fraught.

\section{(Re)using Qualitative Data? Questioning Key Terms and Assumptions}

2.1 The development of specific discussions about 'reusing qualitative data' or 'secondary analysis of data' relies on the assumption that 'reusing' qualitative data is different to 'using' qualitative data, and thus requires a new set of debates and discussions. This is based on a further assumption, namely that secondary analysis depends on an object which has been termed 'secondary data', and which is therefore distinguished from 'primary data'. Questioning these assumptions poses a further question: do multiples turns to, and returns to, qualitative data constitute something 'new', to be termed reuse of data, or secondary analysis of data?

2.2 For some, secondary analysis of qualitative data constitutes a new kind of methodology, which is required because secondary data is seen to constitute a new type of data (Heaton 2004a). However, I suggest that it is the conception of secondary analysis of qualitative data as new, that requires that an object, 'secondary data', be brought into view, and distinguished from other kinds of data. We need to ask how is this data made to be secondary data; and how this approach becomes a new methodology - not least as there are any number of instances in which something akin to reuse of qualitative data already happens, such as the practice of returning to data collected earlier in an academic career; or the sharing of interview material in research teams. Elizabeth Silva even suggests that 'processes of reusing data are quite routine in social sciences' (Silva 2007: para 1.2). There are a number of ways in which this is achieved: by establishing a number of boundaries, most notably between primary and secondary data, and primary and secondary analysis; between secondary analysis of quantitative data, and secondary analysis of qualitative data; between the use of secondary data in different disciplines, particularly, history and sociology; between different archives, such as ESDS Qualidata and Mass Observation. Yet the consequences of producing secondary analysis of qualitative data as a new methodology are that we are left bereft of any connections with existing methodologies which might offer some insight into the question of how to go about this kind of research.

\section{Pre-existing Data: Primary and Secondary Data}

2.3 Reuse poses a chicken and egg question: which comes first, secondary analysis, or secondary data? Janet Heaton suggests in her book, Reworking Qualitative Data, that the 'the first and most rudimentary principle of secondary analysis is that it involves the use of pre-existing data' (Heaton 2004a: 2). Yet the notion of pre-existing data is at odds with the widely held understanding in qualitative research that data is not collected, gathered or found, but rather that it is created and co-produced in the research process, between researcher and respondent (Mason 2002, Law 2004 for example). Understanding data as preexisting fails to attend to the complexities of how data is co-constructed through a new research project. A new research project provides a new context for the creation and emergence of 'data', particularly through the contemporary production of the relationship between researcher and data. Thus secondary analysis is 
not so much the analysis of pre-existing data; rather secondary analysis involves the process of recontextualising, and reconstructing, data. Once the data is transformed through the process of recontextualisation, it is not so much that we now have a new entity to be termed 'secondary data', and which might require a new methodology to be termed secondary analysis, rather, that through recontextualisation, the order of the data has been transformed, thus secondary analysis is perhaps more usefully rendered as primary analysis of a different order of data, as accounts of reuse by Bishop (Bishop 2005b), Savage (Savage 2007) and Silva (Silva 2007) so clearly demonstrate.

\section{Linking Data and Methodology}

2.4 Heaton's construction of secondary analysis as a new methodology relies on two assumptions. Firstly, Heaton links data type and methodology, that is, rather than linking research questions with methodology and analysis of data (Mason 2002). For Heaton it is the type of data which determines questions of methodology; so for Heaton secondary data is a new kind of data which therefore requires a new kind of methodology. Secondly, in claiming secondary analysis of qualitative data as a new kind of methodology, Heaton is forced to find ways to dismiss and discount what we might think of as other kinds of secondary analysis. While it is true that methodology and data are linked, we might more usually link the research questions we are seeking to explore with the methodology which we choose. That is, while it is clearly not possible to use multiple regression or visual analysis in interpreting the text of an interview, we might more usually be thinking about choosing between different kinds of discourse analysis, narrative analysis, content analysis, for example, and the decision about which of these to choose is likely to be related to research questions and the epistemology and the ontology of the researcher (as well of course as disciplinary background, and particular training and predilections of the researcher) (Mason 2002). Furthermore, because Heaton so clearly links methodology and data, the need to distinguish between different methodologies also requires distinguishing between different kinds of data.

2.5 In constructing secondary qualitative data as distinct from other kinds of data, which might also be considered as kinds of secondary qualitative data, Heaton draws on the distinction between naturalistic and non-naturalistic data. Here 'naturalistic' data are data which are ' "found" or collected with minimal structuring by researchers' and ' "non-naturalistic" data are data that 'were solicited by researchers'. Examples of naturalistic data include life stories, autobiographies, found diaries, letters, official documents, photographs, film and social interaction. Examples of non-naturalistic data include fieldnotes, observational records, interviews, focus groups, questionnaires, solicited diaries and life stories (Heaton 2004a: 5). Heaton reserves secondary analysis for interpreting non-naturalistic, that is, artefactual data, and documentary analysis and conversation analysis for the interpretation of naturalistic data, which is found or collected with minimal interference by researchers.

2.6 However the use of the terminology, naturalistic and non-naturalistic, is unfortunate. While there may be benefits to maintaining these distinctions, the term naturalistic (and non-naturalistic) risks underplaying the work and artifice involved in the production of any story, even and perhaps especially stories which appear 'untouched' by the researcher. ${ }^{[2]}$ Stories which emerge in everyday life may exist, and be produced in the context of, a variety of circuits of meaning, which may include personal and familiar references, media and popular culture, and even academic discourses as these might circulate in contemporary culture. This term and distinction risks naturalising some stories, or more precisely the conditions of production of some stories; and can fail to attend to what happens to a text when it moves from being a 'naturalistic' diary, autobiography, photograph or whatever and comes into the context of a research project.

2.7 A further way in which this rupture between primary and secondary data/analysis is produced is through comparisons between secondary analysis of qualitative and quantitative data.

\section{Comparing Qualitative and Quantitative Secondary Data}

2.8 Discussions of archiving and re-using qualitative data almost inevitably take quantitative data as a point of comparison. For some, turning to secondary analysis of qualitative data is presented as a development of traditions of secondary analysis of quantitative data. Janet Heaton cites Barney Glaser (more renowned for his work on grounded theory) from 1962, as 'one of the first analysts to recognise the potential of reusing qualitative research studies' (Heaton 2004a: vii), and who suggests that 'secondary analysis is not limited to quantitative data' (Heaton 2004a: viii). She also cites Angela Dale, '... that there is 'no reason why statistical data should be the only material available for secondary analysis' (Dale et al 1998: 15 cited in Heaton 2004a: viii). Heaton presents an evolutionary model in her chapter 'From Quantitative to Qualitative Secondary Analysis', with secondary analysis of qualitative data 'behind' quantitative analysis: '[f]our decades later, however, secondary analysis remains ill-defined and underdeveloped as a qualitative methodology' (Heaton 2004a: vii). 
2.9 However, the purposes of comparisons with secondary use of quantitative data vary. While for some, such as Heaton, the comparison serves to suggest the potential of secondary analysis of qualitative data, and as an argument for further attention to the problems and promises of reusing qualitative data, for others the comparison is invoked precisely as evidence of the incommensurability of the data. This radical difference of the data is then the grounds for arguing the distinction between re-using qualitative and quantitative data, and related issues of archiving data, or even suggestive of the limitations of re-using qualitative data. The Boddy Report for the ESRC on data archiving noted 'considerable concerns within the research community over the archiving and re-use of qualitative data', and concluded that 'the argument that qualitative data can be treated in terms of archiving policy and practice on an equivalent basis to quantitative data is not commonly accepted' (2001: 37). For Parry and Mauthner, it is the difference between quantitative and qualitative data (and importantly the differences in how that data is gathered, what counts as data and what gets archived, or not) that, in their view, renders secondary analysis of qualitative data so problematic: '[w]e reiterate concern with the assumption that archived qualitative data can be used to generate new substantive findings and theories, in an equivalent way to quantitative data' (Parry and Mauthner, 2005:141).

2.10 However, I am less concerned here with how secondary quantitative data are invoked for different ends, than with paying attention to how quantitative data are presented as the natural and inevitable point of comparison for secondary qualitative studies, how quantitative data analysis remains the inevitable point of reference for evaluating research, obvious from the still ubiquitous requests for qualitative researchers to explain and justify 'non-representative samples', 'small samples', issues around validity, representativeness, generalisability etc. Quantitative data remain hegemonic within sociological research, not least because quantitative evidence 'fits' within a particular conception of how data can be stored, distributed and valued in neo-liberal cultures characterised by neo-managerialism and an audit culture.

2.11 Such a comparison may be understood to serve a strategic purpose in legitimising a process which is still viewed with some suspicion. In this light, the support of key figures in the world of methodology, such as Barney Glaser and Angela Dale, is not insignificant. Yet there are limitations to this strategic move. Judith Butler has suggested that 'strategies always have meanings that exceed the purposes for which they are intended' (Butler 1990: 4). The strategy of comparing secondary qualitative and quantitative research, relies on a number of assumptions, which I suggest undo and undermine the possibilities of reusing qualitative data, rather than supporting it.

2.12 It is in the work of Mike Savage (Savage 2007) that we can begin to grasp the potential of drawing on the reuse of quantitative data to think through the possibilities of reusing qualitative data. Notably for Savage the possibilities of qualitative data reuse rely less on any strategic comparisons with quantitative data or methodology, than with careful attention to the nature of the research questions which the reuse of quantitative data is used to answer. Savage's recognition that quantitative data from different historical moments are often used to make claims about trends over time, offers him a way of thinking about the possibilities of reusing qualitative data to examine socio-cultural change. This kind of creativity about the possibilities of qualitative data reuse is undermined rather than enabled by more mechanistic efforts to compare qualitative and quantitative data.

2.13 Furthermore questioning the assumption that quantitative data are a necessary point of comparison for secondary qualitative studies reveals how this comparison eclipses other possible comparisons. For those who suggest the possibilities of extending secondary analysis from quantitative to qualitative data there is nonetheless little in the literature on reusing quantitative data which will help with thinking through the issues raised by qualitative data, requiring the construction of a new field apparently from scratch. The potential for drawing on existing tools and methodologies which qualitative researchers have developed for working through 'primary' data, such as documentary analysis, conversational analysis sources, or oral histories; other disciplines such as history which have an extensive tradition of archiving and analysing documents; and archives other than ESDS Qualidata, such as the Mass Observation Archive; is neglected. References such as that by Hammersley to the benefits of a turn to history are rare and not fully developed in this literature. Hammersley, in an article on 'Qualitative Data Archiving: Some Reflections on its Prospects and Promises', cites Lawrence Stenhouse, who proposed a qualitative data archive as early as 1978 , on history as the appropriate point of comparison for sociology (than natural science) and that such an archive would be equivalent in function to archives of historical documents (1997: 134).

\section{Disciplinary Boundaries}

2.14 Other comparisons are possible when thinking through the possibilities of re-using qualitative data, such as with history, or oral history, or even literature. Oral history offers a particularly useful point of comparison as it is perhaps one point where sociology and history connect most, although this is contrary to accounts in the literature on reuse which works hard to establish a considerable difference between oral 
history and sociological interviews, a difference which is surprising given the ease with which quantitative data are taken as a point of reference.

2.15 For Janet Heaton, oral history archives are yet another instance of naturalistic data, which for her renders the data different to qualitative interviews generated in a sociological context (Heaton 2004a). Parry and Mauthner do mention oral history, but distinguish oral history from social science on a number of grounds: that 'oral historians are the only qualitative researchers who archive their data as a matter of course'; and that 'whereas within oral history a main purpose of data collection is to secure an historical record for future and current access, social science data are seen mainly as a potential resource to generate new hypotheses, findings and theories' (2004: 148). However, these distinctions do not hold; not all oral historians archive their data; and distinctions between oral historians and social scientists and their intellectual pursuits are not always so neat. Some social scientists use oral history in their research precisely to generate hypotheses, findings and theories (for example Brodkin Sacks 1989, Ginsberg 1989); it is possible to be an oral historian and a social scientist, these are not necessarily mutually exclusive categories. While Parry and Mauthner distinguish social science and oral history research as different disciplinary locations, it is worth recalling the origins of QUALIDATA, the body which has given rise to many of these discussions. Here distinctions between social science and (oral) history are much more difficult to maintain: QUALIDATA emerged in part from the vision of Paul Thompson, oral historian in the Department of Sociology at the University of Essex.

2.16 Rather than make these distinctions between social science and oral history as different forms of qualitative research, it might be more productive to examine what the theory and practice of oral history can offer other qualitative researchers (sociologists and beyond) who want to think through the implications of archiving data and/or the process of drawing on existing oral histories, interview material and other research data. This would suggest that contra Parry and Mauthner's urge to indicate hard and fast boundaries between oral history and social sciences, and the need to develop discipline specific guidelines, that the future of 'reusing qualitative data' might be more successfully assured, not by insisting on disciplinary distinctions, but by breaching disciplinary boundaries. This may reveal the productive possibilities of different disciplinary engagements with the issue of archiving and using data from archives (and this includes not just oral history and history, but also art history, museology and so on).

2.17 Furthermore the almost exclusive focus on ESDS Qualidata in the literature neglects attention to another important archive, the Mass Observation Archive (MOA <http://www.massobs.org.uk>) at the University of Sussex. The Mass Observation Archive, a project begun in 1937, is arguably one of the largest collections of qualitative data in the UK. The current Mass Observation Project has been in existence since the early 1980s, offering unique opportunities for qualitative longitudinal research. While ESDS Qualidata mainly archives 'primary' research projects which are deemed of possible future interest to further researchers, the current Mass Observation Project has collected data since 1981 which is mainly intended for 'secondary use'. Approximately three times a year, questionnaires, called 'directives', are sent to volunteer writers around the UK. Directives usually consist of a number of questions on topics of contemporary interest, ranging from adultery, to the death of Princess Diana, to recent general elections, and 'correspondents', as the Mass Observation (MO) participants are known, are asked to write back with their thoughts, opinions and experiences. Thus, returning to earlier concerns, MO raises interesting issues here: about whether MO data can best be understood as primary or secondary data; and whether using MO data involves primary use or secondary reuse; whether data can be reused, if they have never been used. The Mass Observation Archive acutely throws into question many of the premises of debates about reusing qualitative data, at the same time as it points to the neglect of a rich history of grappling with the dilemmas of using qualitative data generated in a different context (Bloome, Sheridan et al. 1993, Sheridan 2000, Sheridan, Bloome et al. 2000, Hubble 2005, Koa Wing 2005).

2.18 As well as disregarding oral history, ignoring other possible sources such as the MOA, distinctions between qualitative and quantitative data rely on characteristics of qualitative data to establish the incommensurability of such data. The features of qualitative research most invoked are the importance of context in the construction of qualitative data, and the role that reflexivity plays in the research process. Thus 'context' and 'reflexivity' are understood to mark the boundary between quantitative and qualitative data, and it is the apparent impossibility of archiving context and reflexivity which renders qualitative data problematic to reuse, in these accounts. The following section turns to examine how context and reflexivity have been invoked.

\section{Reinterpreting Principles of Qualitative Research}

Context ${ }^{[3]}$ 3.1 As mentioned earlier, one of the most persistent arguments around re-using qualitative data is about whether it is possible to reuse data outside of the original context in which it was collected, or without sufficient contextual data. Intrinsic to this argument is the understanding that meaning making in 
qualitative research emerges out of the reflexive interpretative practices of the researcher. As Heaton writes: '[i]n qualitative research, the interpretation of data is generally perceived to be dependent on the primary researcher's direct knowledge of the context of data collection and analysis obtained through their own personal involvement in the research' (Heaton 2004a: 30).

3.2 Those linked with ESDS Qualidata have been particularly responsive to this issue, and have suggested and put in place procedures to improve documentation of the research process in the course of archiving qualitative research data. However some do not believe that the archiving of more contextual information will render qualitative data more usable. Mauthner et al. (1998) argue that criticisms of archiving qualitative data have rested on the assumption that without this background information, the data remains incomplete; and that the suggested solutions of making sure that as much information as possible is archived with the data implies that this will then produce a complete dataset. They are critical of the realist and positivist ontology and epistemology underlying this model of archiving and re-using data, and the 'practical' solutions offered to address this issue, which they assert cannot adequately work through the implications of the reflexive construction of qualitative data. Implicitly they are arguing that reflexivity cannot be archived, that this reflexivity is essential in order to reuse data and therefore reusing qualitative data is problematic. Drawing on guidelines from the QUALIDATA website (and discussions of these issues in Hammersley 1997), which stress QUALIDATA's commitment to ensuring 'sufficient documentation of the research proposal, aims, methods and outcomes to enable reuse' (QUALIDATA 1997: 7, cited in Mauthner et al 1998: 735), they suggest that these guidelines imply that these measures 'will restore the data to the status that they had for the primary researchers and enable them to be used to generate new findings or theories' (735). Though it is worth noting that QUALIDATA settle for the more modest goal of 'sufficient to enable reuse'. For Mauthner et al. these are largely practical solutions to what is for them an epistemological problem about the nature of qualitative research. Thus they are critical of Hammersley. Although they suggest he does recognise the epistemological nature of some of these issues, for them he poses insufficiently philosophical solutions:

If researchers generate new substantive findings and theories from old qualitative data, without attending to the epistemological issues, they are being naively realist thus unwittingly serving to reify the data by hoodwinking us into believing they are entities without concomitant relations. Furthermore, while archives may be an extremely rich source for historical and methodological exploration, any attempt to go further than this is incompatible with an interpretive and reflexive epistemology. Archived data clearly have value as historical documents for studying the past. Methodologically, they can provide insights about where and how researchers were positioned in relation to theoretical, epistemological, methodological and substantive issues of the time of the research. (Mauthner et al. 1998: 743)

3.3 However, the account of Mauthner and colleagues, despite claims to being interpretivist, is almost as realist as those they critique, in their implication that because it is not possible to archive everything, including the reflexive production of data, that the possibilities of meaning-making from secondary data are limited - here, somewhat disparagingly to 'history' and 'methodology'. This account of history, and sociology, proceeds as if historical knowledge can be separated from social science knowledge, after all the social sciences too exist in historical time, and historians are often excellent in being reflexive. Yet Mike Savage's account of using the Mass Observation Archive offers a much more complex account of the possibilities of reusing data, and of the significance of history and methodology (Savage 2007; see also Bornat 2003). Rather than focusing on how one particular study could be read, Savage looks at a number of studies in the MOA on class identities to examine changing understandings of class over time. His work draws not only on respondents' responses to questions about class, but also on details of the research process itself as data, as something to be examined as part of the research process, rather than as a pregiven 'context' with which to make sense of the 'data'. For Savage the ways in which researchers sought to elicit class identities were as valuable data as respondents' commentaries.

3.4 However, for Mauthner et al. it is not only the latent realism in their account that gives rise to such an impoverished notion of the possibilities of reusing data; their construction of 'reflexivity' is also implicated in their account of the problems of reusing qualitative data. The following section raises two problems with their account of reflexivity, and the work which reflexivity is used to do.

3.5 Mauthner et al. ask percipiently (in the title of their article) 'the data indeed are out there, or are they?' and they seem to conclude that the data are indeed out there, just possibly not of much use, because 'out there' is constituted as so far in the past that the data can only be used for some impoverished historical or methodological research, and that the data cannot be used to generate substantive new research findings. Arguably, however, it is the rider to their question that is important here - 'or are they?'. Bearing in mind my earlier questioning of the notion of pre-existing data, I suggest that the data in fact are not 'out there' at all, that the data are here and now, being constructed in the process of a new research project (Savage 2007, 
Bishop 2005b, Silva 2007 all demonstrate this clearly). A central problem then in the account of Mauthner and colleagues is their failure to attend sufficiently to the contemporary context and reflexivity of the current, 'new', research project. Their account, with its attention to the context and reflexivity involved in the production of the so-called 'pre-existing' data, proceeds at times as if they understand reusing data to be about some attempt to reconstruct the original research project. They fail to appreciate the necessity of attention to the context and reflexivity of the current project, which effectively makes new data out of old. Ironically it is their mistaking of the temporality of the context and reflexive production of the data which leads to their account of the limitations of re-using data. Their construction of the issues in this debate consistently leaves the data behind in the past, in the original project that produced the data. This highlights a further limitation of terminology such as 'secondary analysis', which emphasises the process of 'analysis', at the expense of attention to the setting up of and the production of the current research project. As Libby Bishop notes, 'the implication is that archived data are immune from practices of coconstruction and reflexivity' (Bishop 2005b). The following section explores the consequences of this deployment of reflexivity, and the mistaking of the temporality of the research process, further.

\section{Reflexivity}

3.6 For Mauthner et al. reflexivity is invoked to signal a shift from practical to epistemological concerns, in their efforts to shift the terrain of the debate from 'practical' solutions to recognition of the epistemological issues that the question of background information raises (1998). Their primary argument is that qualitative data is reflexively produced, that the data is a co-construction of the researcher and the research participant. They argue that the epistemological implications of the reflexive nature of the production of qualitative research have not generally been adequately explored in secondary analyses: '[e]pistemological concern has previously been expressed about the use of archived data for substantive and theoretical inquiry. It has been argued that because the conditions under which these data are produced are inescapable, their reinterpretation at some later date is rendered problematic' (Parry and Mauthner 2004: 141).

3.7 For Mauthner et al. reflexivity distinguishes qualitative data from quantitative data (1998). However this account of quantitative and qualitative research relies on accepting discourses of the objectivity of quantitative research at face value. While it is of course qualitative research that has foregrounded questions of the situatedness of knowledge, contrary to the 'god trick of seeing everything from nowhere' (Haraway 1988: 571), the comparison, perhaps unwittingly, upholds the notion of the objectivity of quantitative research. While researchers may commonly behave as if quantitative data are objective, ultimately, all data, qualitative and quantitative, are 'situated knowledges' which can only be understood as and through 'partial perspectives'. The distinction is not so much that qualitative data are situated and context bound and quantitative are not, but that reflexivity reveals all research qualitative and quantitative as situated; however the qualitative researcher is more likely to open up the process of knowledge production to scrutiny, and to attend to the conditions of production of the research.

3.8 Drawing on work by Adkins, I want to follow through the implications of this use of reflexivity. Adkins writes that 'reflexivity enacted at an epistemological level has been understood to undo the conventions of dominant ways of knowing and provide a ground for the creation of alternative knowledges' (Adkins 2002: 85). However she suggests that 'such reflexivity should not be read as transgressive but rather as involving the very inscription of difference and the making of hierarchies' (Adkins 2002: 84). This argument by Adkins provides a useful way of reading the work which Mauthner et al. use 'reflexivity' to do. Their efforts to insist on the impossibility of archiving reflexivity, rely on the 'inscription of difference and the making of hierarchies'. For Adkins self-consciousness on the part of reflexive ethnographers often relies on making respondents as well as 'the field' stationary (Adkins 2002: 345). In order for the reflexive self of the researcher to appear, a number of subjects are fixed: here, the earlier self of the researcher, the research respondents, and the data. Adkins points towards 'the ways in which such reflexivity enacts not so much subject-subject relations but a configuration of the relation between subjectivity and knowledge or knower and known which only allows certain subjects to speak' (Adkins 2002: 85-86). Specifically Adkins argues 'that reflexivity in relation to knowledge practices concerns a speaking position constituted in terms of a mobile relation to identity on the side of the knower in relation to the known, a position from which there are a number of exclusions' (Adkins 2002: 86). Mauthner et al. consistently fix the data in the past, fix disciplines at particular moments, fix the earlier self of the researcher as naïve, and fix respondents also. All are rendered immobile, in a way which enables the researcher to have moved, and to produce herself as a more sophisticated academic:

We had not only moved on in our personal lives, but also in the disciplines in which we worked, in our substantive interests and in our theoretical and methodological positionings. (Mauthner et al. 1998: 739) 
In particular we found our data to be constrained both by the concepts and ideas which were current at the time of our research, not only in the academic world but also in our own worlds and the worlds of the respondents. (Mauthner et al. 1998: 741)

As a consequence the data were produced reflexively via interaction between the researcher and respondents through the very act and experience of participation. The data were inextricably bound to the unique conditions of their production. While interesting from a methodological stand-point, for the purpose of generating either new substantive findings or theories the data were wholly inadequate. (Mauthner et al. 1998: 740 )

3.9 This fixing, of data, respondents and disciplinary knowledges, enables Mauthner et al. to provide accounts of their researcher selves which highlight their mobility, academically and personally, in terms of disciplinary knowledges, epistemologies and methodologies. Through this demonstration of academic professionalism, the possibilities of reuse are discounted as not meeting rigorous commitments to the principles of qualitative research such as attention to context and the reflexive production of data and knowledge claims.

2.10 This implicit allusion to the institutional context for debates about archiving and reuse begins to offers some suggestions about why this debate has become so heated, and I turn to this in the conclusion.

\section{Conclusion}

4.1 To return to my opening argument, questioning the distinction between reuse and use of data. My point is not to suggest that research which has come under this rubric does not require careful methodological consideration, that some 'new' issues are not brought into being. In questioning whether we need something 'new' called 'secondary data analysis', and whether and how this could ever be considered a new methodology, my intention is to suggest more a promising future for re-using qualitative data than some of the more sceptical accounts might currently imply. Accounts of secondary analysis of qualitative data as a new methodology require the forgetting of a host of possible resources for thinking through the implications of reusing data, from archives such as the Mass Observation Archive at Sussex, to the traditions of documentary analysis, and other methodologies drawn on by historians, to the analytic techniques already developed by qualitative researchers. Hammersley, even in 1984, was talking about the 'paradigmitis' that afflicted qualitative work, 'the tendency to be constantly inventing "new" approaches, or at least preoccupied with defending one approach against each other' (Hammersley 2004: 26). Artificial boundaries, between primary and secondary data, between primary and secondary analysis, between qualitative and quantitative research, between sociology and history / oral history, between documentary analysis and secondary analysis, both reduce the complexity of the issue, and at the same time, increase complexity, by positing an absence of a history of reusing data, and insisting on the need to invent from scratch.

4.2 Yet, while suggesting that reuse of qualitative data is not quite new, nonetheless there maybe something 'new' which is giving rise to some debate. Yet if there is something new about all of this, it is not the reuse of qualitative data per se, rather it is the injunction to archive and the bureaucratisation and institutionalisation of this process. What is new is a changing academic context for discussions of reuse of qualitative data. So despite the almost obsessive attention to context in the literature on reuse, there has been little attention to the institutional context within which this debate has emerged.

4.3 The emergence of QUALIDATA has forced questions of reusing data on the social science agenda in a new way. It is not that academics were not reusing data prior to the setting up of QUALIDATA. But the emergence of QUALIDATA has provided a particular focus for researchers' concerns. What was new, to some social scientists, was the need for ESRC grant applicants (i) to demonstrate that data did not already exist which would address the stated research questions and (ii) to offer one's data, and possibly other documents and notes associated with the research, for archiving. While the former has not provoked much controversy, the latter certainly has. A number of accounts suggest rather straightforwardly that researchers have little to worry about, either about depositing transcripts of interviews, or about depositing field notes. Glaser wrote that, '[i]n fact, some field workers may be delighted to have their notes, long buried in their files, reanalyzed from another point of view' (Glaser 1962: 74). Similarly Paul Thompson noted of his own data from The Edwardians study:

It was incredible how many other researchers came to use it. At least five times as many major publications came out of it as the original research team could have produced. That has been an enormous source of satisfaction to me as a researcher. I want to encourage anyone here who has not yet deposited, that it will give you great pleasure and pride in the longer run, to have your work used in that way too. (Thompson 2004: 83-84) 
4.4 These endorsements of reuse are in the public domain, and the tenor of these accounts suggests a response to an implied researcher reluctant or even overtly resistant to archiving and reuse. Yet accounts of strategies of resistance from researchers who do not wish their ESRC funded data to be archived tend to be anecdotal, for example, emphasising to research participants that they do not have to give their consent for their interviews and/or transcripts to be archived. Discussions about reusing qualitative data rarely comment on the emergence of QUALIDATA at a time of radical change in higher education in the UK, a time of a turn to neo-managerialism and audit culture, and an emphasis on cost-effectiveness and value for money (see for example Shore and Wright 1999). It is in this context that the ESRC began funding QUALIDATA in 1994, and that ESRC research grants have been understood to be 'public' money, with the implication that because it is 'public' money, academics should be held more accountable. These discourses form part of the context for the emergence of QUALIDATA, and I suggest part of the context for some academics resistances to archiving and sharing data. This is a radically different context to that sketched by Paul Thompson, commonly regarded as a founding father of oral history research in the UK. Thompson has written about the emergence of QUALIDATA from his oral history project which led to the book The Edwardians:

The experience of this project was the origin of the idea for creating a national archive for fieldwork data, which led to the setting up of Qualidata in 1994. It was equally the seedbed for my own subsequent belief in the crucial potential of secondary analysis in qualitative research (Thompson 2000). We very quickly realized that our interview material could be valuable for far more people than ourselves, and we were able to use a store cupboard in the Department of Sociology [at the University of Essex] for it. We created the Oral History Archive there.' (Thompson 2004:83-84)

4.5 Thompson's vision of an altruistic culture of sharing among academic colleagues serves as a reminder of a different academic culture. This more utopian, grassroots account of the emergence of QUALIDATA, appears in tension with the story of the ESRC's growing interest in cost-effectiveness, but is perhaps more an indication of a very particular moment of change in academia in the UK, when new ways of organising and organising academic work were being introduced. However this tension is important to note, because it may in part account for some academics' resistance to the imperative to archive: that it so quickly got entwined with the more functionalist reform of higher education and all the associations we have with this, of audit trails, bureaucratisation of academic life, and increasing pressures to produce.

4.6 Colin Bell suggests some of the anxieties which some sociologists may have about laying bare their research, and indeed, links his reflections with discussions about depositing material in QUALIDATA (Bell 2004: 33). Bell reflects on what he calls 'owning up accounts', and his efforts to produce the book subsequently published as Doing Sociological Research, and to find contributors, 'But we found it quite difficult to trace all the right people, or get people to agree. A lot of people thought this was absolutely not the right way to behave. You can't reveal what's gone on, you can't write these kinds of accounts.' (Bell 2004: 31). While Bell doesn't elaborate on why exactly people thought these kinds of accounts couldn't be written, the way he presents these stories suggests that the 'why' is self-evident - people build their academic careers on neat/clean/rational accounts of the research process, construct themselves as competent and expert, and to reveal one's fallibility, in public, in writing, is not the done thing. Doing Sociological Research was then one of the first collections of first person accounts of the research process, which attempted to present the process of research 'warts and all'. Bell, understandably pleased that the first person accounts revealed that findings produced in the process of the research were the result of considerable negotiations, wrote,

\footnotetext{
...we genuinely believed, in the naïve, heady mid-seventies, that you could have an annual Sociological Research Yearbook, which you would get everyone who had published that year to own up - there could be surveys of methodological innovations or whatever. Maybe we could have done it as a journal, but we could not get anybody to buy into it at all. ... Concluding then, it is not a genre that has thrived. ... This is not an unreasonable genre. But it has almost died. (Bell 2004: 33)
}

4.7 Issues of professionalism remain significantly undertheorised in the literature on methodology. Research, when written up, is not only a report on research, but is also the construction of an academic career (see Silva 2007 for an elaboration of this). There is a fundamental unevenness in the research process where the interviewee is accorded anonymity but where by definition the researcher is made visible in the process of constructing a professional identity, and this context then means that it can be difficult to reflect publicly on what is said and not said and written and not written about the research process. This means then that there may be aspects of the research process which the researcher feels are key to the research experience, and to their interpretation of the research which they are unwilling to document, and very unwilling to place in an archive. As Nairn et al. (2005) noted recently, suggesting that despite a decade 
or more of reflexivity, that it has become no easier to recount the dilemmas of research: 'To place a 'failed' interview at the centre of an academic article is risky and exposing. After all, the academic arena, is a competitive one where 'success', rather than 'failure', is rewarded.' Libby Bishop begins her paper on reuse by noting her own fears from her research diary, and observes that 'the craft of research involves thinking and exposing how that is done can be unnerving' (Bishop 2005b). The literature on re-using data has more generally been concerned with ethical issues of confidentiality and anonymity with respect to research subjects. Yet, with the turn to reflexivity in research, distinctions between the researched and the researcher are less clear; reflexivity also involves the researcher as a 'subject' of research. However the ethical issues around this remain undertheorised; and the lack of attention here contributes to reluctance to deposit data and the difficulties and challenges of revealing how one's mind works - and perhaps even more scarily one's emotions about research, and how these impinge on the research process, on what is said and written about the research process and what is, or is not, deposited in archives. Thus while concerns about the problematics of archiving and reusing data are presented in terms of the 'professional' academic's concerns about the methodology, epistemology and ethics of research, there is little explicit attention to how the construction of a professional academic career impacts on debates about reusing data.

4.8 Though not writing specifically of 'reusing qualitative data', John Law's book, After Method: Mess in Social Science Research, is nonetheless pertinent, not least given my suggestion that much that is appropriate for 'primary' qualitative research is equally appropriate for 'secondary' research (Law 2004). Law advocates attention to the 'mess' of social science research, noting that 'more often social (and still more natural) science "method talk" connotes something quite different - that is a particular version of rigour' (Law 2004: 9). Though he is aware that in order to do this :

we will need to unmake many of our methodological habits, including: the desire for certainty; the expectation that we can usually arrive at more or less stable conclusions about the ways things really are; the belief that as social scientists we have special insights that allow us to see further than others into certain parts of social reality; and the expectations of generality that are wrapped up in what is often called 'universalism'. But first of all we need to unmake our desire and expectation for security. (Law 2004: 9)

Eschewing our comfort zones, and developing a more creative, and even messy, approach may be the key to opening up the full potential of qualitative data reuse.

\section{Notes}

${ }^{1}$ This paper, and those by Bishop, Mason and Savage, were first presented at a workshop on 'Reusing Qualitative Data' organised by the Centre for Research on Socio-Cultural Change (CRESC) at the University of Manchester on 28 September 2005. One of CRESC's innovations as an ESRC-funded Research Centre has been attention to methodology as a substantive topic. This focus has driven the creation of a Qualitative Research Laboratory (Niamh Moore), and a Cultural Statistics Laboratory (Shinobu Majima). Given CRESC's focus on examining claims about socio-cultural change, and a commitment to theoretically informed empirical research, there is a particular interest in looking at diverse sources of data, and thinking about how these may be (re)used most appropriately. One exciting element of the workshop was bringing together speakers from key UK archives of qualitative data, Libby Bishop from ESDS Qualidata and Sandra Koa Wing from the Mass Observation Archive. Sadly, Sandra has recently died and will be greatly missed. Her book, Mass Observation: Britain in the Second World War, will shortly be published by The Folio Society. As well as thanking other presenters, and participants, I would also like to thank Jeannette Edwards, Nick Hubble, Elizabeth Silva and Corinne Squire for acting as discussants on the day. See <http://www.cresc.ac.uk/events/archived/methodsworkshop.html> for an account of the workshop.

2 See also Silva (2007) for an account of reusing qualitative data and the tensions created by a naturalistic approach.

${ }^{3}$ See Moore (2006) for a more extended account of the problem of context in debates about reusing qualitative data, and specifically the lack of attention to the theorisation of context in literary theory and cultural studies, and what might be gained by turning to such accounts.

\section{References}

ADKINS, L. (2002) 'Reflexivity and the Politics of Knowledge' in Revisions: Gender and Sexuality in Late Modernity, Buckingham: Open University Press. 
Research Methodology, Vol. 7, No. 1, pp. 29 - 33.

BERGMAN, M. and T. S. Eberle (2005, May) "Special Issue: Qualitative Inquiry: Research, Archiving, Reuse." Forum Qualitative Socialforschung / Qualitative Research Forum [On-line Journal] Volume, DOI.

BISHOP, L. (2005a) 'Protecting Respondents and Enabling Data Sharing: Reply to Parry and Mauthner', Sociology, Vol. 39, No. 2, pp. 333 - 336.

BISHOP, L. (2005b) 'Is Secondary Analysis Second Best? A Case Study of Reusing Qualitative Data', Paper Presented at the CRESC Methods Workshop: Reusing Qualitative Data, University of Manchester, 28 September.

BLOOME, D., D. SHERIDAN and B.STREET (1993) Reading Mass Observation Writing: Theoretical and Methodological Issues in Researching the Mass Observation Archive', Mass Observation Archive Occasional Paper No 1. Brighton, Mass Observation Archive, University of Sussex Library.

BODDY, M. (2001) Data Policy and Data Archiving: Report on Consultation for the ESRC Research Resources Board, Bristol: University of Bristol.

BORNAT, J. (2003) 'A Second Take: Revisiting Interviews with a Different Purpose.' Oral History Vol. 31, Nos. 1-2, pp. $47-53$.

BRODKIN SACKS, K. (1989) 'What's a life story got to do with it?', in Personal Narratives Group, Interpreting Women's Lives: Feminist Theory and Personal Narratives, Bloomington: Indiana University Press.

BUTLER, J. (1990) Gender Trouble: Feminism and the Subversion of Identity , London: Routledge.

CORTI, L., J. Foster and P. Thompson. (1995). "Archiving qualitative research data." Social Research Update Issue 10 Retrieved 13 June, 2005, from <http://www.soc.surrey.ac.uk/sru/SRU10.html>.

CORTI, L., S. Kluge, K. Mruck and D. Opitz (2000, December) "Special Issue: Text, Archive Re-analysis." Forum Qualitative Socialforschung / Qualitative Research Forum [On-line Journal] Volume, DOI.

CORTI, L. and P. Thompson (1998). "Are you sitting on your qualitative data? Qualidata's mission." International Journal of Social Research Methodology 1(1): 85-89.

DALE, A., Arber, S. and M. Procter (1998) Doing Secondary Analysis: A Practical Guide, London: Unwin Hyman.

GINSBERG, F. (1989) Contested Lives: The Abortion Debate in an American Community, Berkeley: University of California Press.

GLASER, B. (1962) 'Secondary Analysis: A Strategy for the Use of Knowledge from Research Elsewhere', Social Problems, Vol. 10, No. 1, pp. 70 - 74.

HAMMERSLEY, M. (2004) 'Towards a Usable Past for Qualitative Research', International Journal of Social Research Methodology, Vol. 7, No. 1, pp. 19 - 27.

HAMMERSLEY, M. (1997) 'Qualitative Data Archiving: Some Reflections on its Prospects and Problems', Sociology, Vol. 31, No. 1, pp. 131 - 142.

HARAWAY, D. (1988) 'Situated Knowledges: The Science Question in Feminism and the Privilege of Partial Perspective', Feminist Studies, Vol. 14, No. 3, pp. 575 - 599.

HEATON, J. (2004a) Reworking Qualitative Data, London: Sage.

HEATON, J. (2004b) 'Wandering off the Beaten Track: Re-using Qualitative Data in Doctoral Research.' ESRC Research Methods Festival 2004, <http://www.ccsr.ac.uk/methods/festival2004/programme/Fri/pm/MSSeminar>

HEATON, J. (1998) 'Secondary Analysis of Qualitative Data', Social Research Update Issue 22 Retrieved 13 June, 2005, from <http://www.soc.surrey.ac.uk/sru/SRU22.html>

HUBBLE, N. (2005) Mass Observation and Everyday Life: Theory, Culture, History, Basingstoke: Palgrave MacMillan.

KOA WING, S. (2005) 'Acquiring qualitative data for re-use: issues from Mass Observation', Paper 
presented at the CRESC Methods Workshop: Reusing Qualitative Data, University of Manchester, 28 September.

LAW, J. (2004) After Method: Mess in Social Science Research . London: Routledge.

MASON, J. (2002) Qualitative Interviewing, London: Sage.

MAUTHNER, N. S., O. PARRY, and K.BACKETT-MILBURN (1998) 'The Data are Out There, or are They? Implications for Archiving and Revisiting Qualitative Data', Sociology, Vol. 32, No. 4, pp. 733 - 745.

MOORE, N. (2006) 'The Context of Context: Broadening Perspectives on Reuse', Methodological Innovations Online [Online], 1 (2). Available at <http://sirius.soc.plymouth.ac.uk/ andyp/viewarticle.php? $\mathrm{id}=27>$.

NAIRN, K., J. MUNRO, A.B. SMITH (2005) 'A Counter-narrative of a 'Failed' Interview', Qualitative Research, Vol. 5, No. 2, pp. 221 - 244.

PARRY, O. and N. MAUTHNER (2005) 'Back to Basics: Who Re-uses Qualitative Data and Why?', Sociology, Vol. 39, No. 2, pp. 337 - 342.

PARRY, O. and N. S. MAUTHNER (2004) 'Whose Data Are They Anyway? Practical, Legal and Ethical Issues in Archiving Qualitative Research Data', Sociology, Vol. 38, No. 1, pp. 139 - 152.

QUALIDATA. (1997) 'QUALIDATA: Guidelines for Depositing Qualitative Data'. [online document from <http://www.essex.ac.uk/qualidata> in 1997].

SAVAGE, M. (2007) 'Changing Social Class Identities in Post-War Britain: Perspectives from MassObservation', Sociological Research Online, 12(3), available at $<\mathrm{http}: / / \mathrm{www}$.socresonline.org.uk/12/3/6.html>.

SHERIDAN, D. (2000) 'Reviewing Mass Observation: The Archive and its Researchers', Forum Qualitative Sozialforschung/ Forum: Qualitative Social Research [online journal], Vol. 1, No. 3, Retrieved 10 September 2005, from <http://www.qualitative-research.net.fgs-exte/3-00sheridan-e.htm>

SHERIDAN, D., D. BLOOME and B. STREET (2000) Writing Ourselves: Mass Observation and Literary Practices, Cresskill, NJ: Hampton Press.

SHORE, C. and S. WRIGHT (1999) 'Audit Culture and Anthropology: Neo-Liberalism in British Higher Education', Journal of the Royal Anthropological Institute , Vol. 5, pp. 557 - 575.

SILVA, E. (2007) 'What's [yet] to Be Seen? Re-using Qualitative Data', Sociological Research Online, 12(3) available at <http://www. socresonline.org.uk/12/3/4.html>.

THOMPSON, P. (2004) 'Pioneering the Life Story Method', International Journal of Social Research Methodology, Vol. 7, No. 1, pp. 81 - 84. 